\title{
ZETA FUNCTION REGULARIZATION OF INFRARED DIVERGENCES IN BOSE-EINSTEIN CONDENSATION
}

\author{
A. M. J. Schakel \\ Institut für Theoretische Physik Freie Universität Berlin \\ Arnimallee 14, 14195 Berlin, Germany
}

(Received January 30, 2003)

\begin{abstract}
A perturbative calculation of the effect of fluctuations in the nonzero frequency modes of a weakly interacting Bose gas on the condensation temperature is reviewed. These dynamic modes, discarded in most of the recent studies, have a temperature-induced energy gap that allows for a perturbative approach. The simple, yet powerful algorithm consists of a high-temperature expansion in conjunction with zeta function regularization of infrared divergences. The algorithm is shown to be reliable by demonstrating that it reproduces known results for a series of examples. With two-loop contributions properly included, the dynamic modes are seen not to lead to a shift in the condensation temperature.
\end{abstract}

Key words: Bose-Einstein condensation, effective field theory, zeta function regularization, infrared divergences.

PACS number(s): 03.75.Fi, 11.10.Wx

\section{INTRODUCTION}

The past five years or so have seen numerous studies aimed to determine the condensation temperature of a weakly interacting Bose gas (for references and critical discussions of the various approaches, see Ref. [1]). These studies, driven by experiments on Bose-Einstein condensation (BEC), were based on a variety of analytic, computational, and experimental methods. With only a few exceptions, however, the theoretical studies took the same starting point of considering only the static part of the quantum field describing the bosons. The rationale is that the dynamic modes have a temperature-induced energy gap. This makes them irrelevant compared to the static mode, for which that gap vanishes, when studying the long-distance critical behaviour of the system close to the phase transition. With the gapped thermal modes decoupled, the microscopic quantum theory reduces to a static, classical theory, assumed to properly describe this thermal phase transition in equilibrium. Universal properties, such as the critical exponents can indeed be computed from this naive theory, as is well known.

Critical temperatures are, however, not universal. Although phase transitions occurring in different systems may display the same long-distance behavior characterized by the same values of the critical exponents, they in general take place at different temperatures. For example, BEC in a relativistic free Bose gas is in the same universality class as BEC in its nonrelativistic counterpart, yet the condensation temperatures differ in both systems [2]. This difference arises because the critical temperatures of these noninteracting systems are determined entirely by the dynamic modes (see Sec. II B) whose spectrum differ for the relativistic and nonrelativistic Bose gases. The universal properties, on the other hand, are determined by the static modes, which are similar for both gases. Whereas universal quantities are largely in- dependent of the dynamics and details of the underlying theory, nonuniversal quantities in principle depend on them. The critical temperatures of lattice models are even sensitive to such short-distance details as the precise lattice used.

The proper way to proceed when calculating nonuniversal properties is well established. One has to go down one level and start from the microscopic model describing the quantum system under consideration and perturbatively integrate out the irrelevant degrees of freedom (the dynamic modes when considering BEC). One thus obtains an effective theory in terms of the remaining, relevant degree of freedom (the static mode in case of BEC), with the coefficients being determined by the irrelevant degrees of freedom that have been integrated out. This effective field program was carried out for the problem at hand almost a decade ago [3], with the conclusion that the dynamic modes lead to a shift in the condensation temperature of a weakly interacting Bose gas. The purpose of this paper is to revise that finding. Namely [4], the shift obtained at one loop is canceled by a two-loop contribution not considered in Ref. [3]. There, only the leading contribution in a high-temperature expansion of the two-loop Feynman diagrams was considered, whereas the canceling terms appear in the next order. To show that it is this omission and not, as has recently been suggested [1], the method used that led to the incorrect conclusion, we clarify and justify in detail the perturbative approach of Ref. [3]. To appreciate the approach, we first recall some essentials of finite-temperature field theory.

\section{A. Finite Temperature}

The properties of a quantum system at finite temperature can be studied [6] by going over to imaginary time 
$t \rightarrow-\mathrm{i} \tau$, with $\tau$ restricted to the interval $0 \leq \tau \leq \hbar \beta$, where $\beta=1 / k_{\mathrm{B}} T$ is the inverse temperature. The time dimension thus becomes compactified and, consequently, the energy variable $\hbar k_{0}$ discrete $k_{0} \rightarrow i \omega_{n}$, with $\omega_{n}$ the so-called Matsubara frequencies,

$$
\hbar \omega_{n}= \begin{cases}2 \pi \beta^{-1} n & \text { for bosons } \\ \pi \beta^{-1}(2 n+1) & \text { for fermions }\end{cases}
$$

where $n$ is a (positive or negative) integer. The difference between the bosonic and fermionic frequencies arises because a bosonic field $\phi(\tau, \mathbf{x})$ satisfies periodic boundary conditions $\phi(\hbar \beta, \mathbf{x})=\phi(0, \mathbf{x})$, whereas a fermionic field satisfies antiperiodic ones $\psi(\hbar \beta, \mathbf{x})=-\psi(0, \mathbf{x})$. Being (anti)periodic, the fields can be expanded in a Fourier series as (with a similar equation for $\psi$ ):

$$
\phi(\tau, \mathbf{x})=\frac{1}{\hbar \beta} \sum_{n=-\infty}^{\infty} \int \frac{d^{d} k}{(2 \pi)^{d}} e^{-i \omega_{n} \tau+i \mathbf{k} \cdot \mathbf{x}} \phi_{n}(\mathbf{k})
$$

where $\phi_{n}(\mathbf{k}):=\phi\left(\omega_{n}, \mathbf{k}\right)$.

An integral over the energy variable $\hbar k_{0}$ at the absolute zero of temperature becomes a sum over the Matsubara frequencies at finite temperature:

$$
\int \frac{d k_{0}}{2 \pi} g\left(k_{0}\right) \rightarrow \frac{i}{\hbar \beta} \sum_{n} g\left(i \omega_{n}\right),
$$

where $g$ is an arbitrary function.

The frequency sums encountered below are typically evaluated using the identity [6]

$$
\frac{1}{\beta} \sum_{n=-\infty}^{\infty} \frac{1}{-i \hbar \omega_{n}+z}=\frac{1}{2}+n(z)
$$

or because of antisymmetry [7]

$$
\frac{1}{\beta} \sum_{n=-\infty}^{\infty} \frac{z}{\hbar^{2} \omega_{n}^{2}+z^{2}}=\frac{1}{2}+n(z)
$$

where $n(z)$ is the Bose-Einstein distribution function

$$
n(z)=\frac{1}{e^{\beta z}-1}=\frac{1}{\beta z}-\frac{1}{2}+\mathcal{O}(\beta z)
$$

The first term at the right hand of the identity (4) gives the result for zero temperature, while the second gives the thermal contribution. Such a splitting is convenient because the pure quantum $T=0$ part can often be combined with the bare (zero-loop) theory. In a renormalizable theory, for example, the zero-temperature part leads to a renormalization of the bare parameters. These contributions are then combined with the bare theory to dress its parameters.
In this paper, the thermal contributions to the grand canonical thermodynamic potential $\Omega$ of various systems are expanded in a high-temperature series. According to standard statistical mechanics, $\Omega$ is related to the partition function $Z$ via [8]

$$
\Omega=-\beta^{-1} \ln Z
$$

Physically, this potential determines the thermodynamic pressure $p$ through $\Omega=-p V$, with $V$ the volume of the system. High-temperature expansions are an important computational tool for studying interacting theories where usually no exact results can be derived as for free theories. The standard approach is to use the identity (4) backwards and to replace the Bose-Einstein distribution function again with an expression involving a frequency sum:

$$
n(z)=\frac{1}{\beta z}-\frac{1}{2}+\frac{2}{\beta} \sum_{n=1}^{\infty} \frac{z}{\hbar^{2} \omega_{n}^{2}+z^{2}},
$$

where in the first term at the right hand, the $n=0$ contribution is isolated. The second term cancels the $T=0$ contribution contained in the sum. These two terms correspond to the first terms in a Laurent expansion of the Bose-Einstein distribution function.

The $n=0$ contribution, stemming from the $\phi_{0}(\mathbf{k})$ mode in the Fourier series (2), is of special interest to us. This zero-frequency mode, specific to bosonic systems, is a thermal mode for which the temperature-induced energy gap $2 \pi \beta^{-1} n$ in the denominator at the left hand of $\mathrm{Eq}$. (4) vanishes. It is related to the static field $\Phi$ alluded to above as follows:

$$
\Phi(\mathbf{x})=\int_{0}^{\hbar \beta} d \tau \phi(\tau, \mathbf{x})=\int \frac{d^{d} k}{(2 \pi)^{d}} e^{i \mathbf{k} \cdot \mathbf{x}} \phi_{0}(\mathbf{k})
$$

where use is made of the Fourier series (2). This classical, zero-frequency mode is the order parameter of BEC in a weakly interacting Bose gas. It develops a nonzero value in the ordered state where the $U(1)$ symmetry $\phi \rightarrow \exp (i \theta) \phi(\theta$ being a constant transformation parameter) is spontaneously broken, with $|\Phi|^{2}$ determining the average number density of condensed particles. That is, a nonzero value of the order parameter (a classical object) implies the presence of a condensate (a quantum phenomenon). Being static, this mode is unaware of the time dimension and only notes the space dimensions. Compared to the dynamic modes $\phi_{n}(\mathbf{k})$, with $n \neq 0$, it lives in one dimension less and its contributions are accordingly different in form from those of the gapped thermal modes, being typical for a $d$-dimensional rather than a $(d+1)$-dimensional theory.

The following hierarchy can be identified, with the pure quantum zero-temperature modes living in $d+1$ noncompact spacetime dimensions, the gapped thermal modes also living in $d+1$ spacetime dimensions, but with a compactified time dimension, and finally the zerofrequency mode living in $d$ space dimensions, which can be understood as $(d+1)$-dimensional spacetime with the 


\section{A. M. J. SCHAKEL}

compact time dimension shrank to a point by letting $\beta \rightarrow 0$. Below, the zero-frequency mode contributions are labeled by a superscript 0 to indicate that they correspond to $\beta=0$, while the zero-temperature $(\beta=\infty)$ contributions are labeled by a superscript $\infty$. The contributions described by the remaining terms in the Laurent expansion (8), finally, are labeled by a superscript $\beta$. We will, somewhat loosely, refer to these last contributions as due to the gapped thermal modes, although they also encode the $T=0$ contributions, that are explicitly subtracted by the second term in the Laurent expansion.

Zero-frequency modes can produce nonanalytic behavior, depending on the number of space dimensions. Dolan and Jackiw [7] in their study of thermal phase transitions in relativistic quantum systems took a pragmatic approach toward these nonanalytic terms induced by such modes and simply discarded them when estimating critical temperatures. In light of the discussion above, this approach is justified as the critical temperature is to first order determined by the vanishing of the quadratic term in the effective theory [9]. Featuring the zero-frequency mode $\Phi$, that theory is obtained by integrating out the gapped thermal modes, which give only analytic contributions. It is only in the next stage, where fluctuations in $\Phi$ are studied, that nonanalytic contributions can appear.

\section{B. Outline}

In this paper, regularization techniques routinely applied to handle ultraviolet divergent integrals are used to stem infrared divergences instead. Usually, such divergences are regularized by introducing an infrared cutoff. This type of regularization forms the infrared counterpart of the momentum cutoff regularization of ultraviolet divergences, where integrals are made finite by integrating only up to a finite momentum. Besides using momentum cutoff regularization, ultraviolet divergences can also be rendered finite by means of dimensional regularization, where the integrals are generalized to arbitrary dimension, or analytic regularization, where powers appearing in the integrands are generalized to take arbitrary values. In the following, these techniques rather than introducing an infrared cutoff are used to regularize infrared divergences.

The infrared divergences in this paper arise when expanding thermodynamic potentials in a hightemperature series, using the algorithm of Ref. [3]. The algorithm differs from the standard high-temperature expansion and focuses on the analytic terms needed for the study of the critical properties of an interacting system. A series of examples is given to show in detail that this high-temperature expansion in conjunction with the regularization techniques used is reliable, by demonstrating that known results are recovered. The examples include a nonrelativistic (Secs. II A and II B) and a relativistic (Sec. II C) free Bose gas, as well as their fermionic counterparts (Secs. III A-III C), and a BCS superconductor (Sec. III D). In Sec. IV A, fluctuations in the nonzero fre- quency modes of a weakly interacting Bose gas are considered by integrating out these gapped thermal modes in a loop expansion. In particular their effect on the condensation temperature is studied using the algorithm advocated here. As mentioned at the beginning of the Introduction, although irrelevant for the critical behaviour itself, these high-energy modes can in principle affect the value of the condensation temperature, which - unlike the critical exponents - is nonuniversal and depends on the details of the underlying quantum theory. Owing to the temperature-induced energy gaps, the contributions due to the nonzero frequency modes can be calculated in perturbation theory. It is shown that the shift in the condensation temperature found in Ref. [3] at one loop is canceled by two-loop contributions [4], not only in $d=3$ but in arbitrary $2<d<4$ (Sec. IV B). The advantage of considering arbitrary dimensions is that $\mathrm{BEC}$ in two dimensions can be studied by taking the limit $d \rightarrow 2$. A discussion of some recent studies on the subject is given in Sec. IV C followed by conclusions in the last section (Sec. V).

\section{BOSONIC INTEGRALS}

\section{A. Bose-Einstein Condensation}

As first bosonic system, a free Bose gas above the condensation temperature is considered. Being noninteracting, the field $\phi_{n}(\mathbf{k})$ describing the bosons can be integrated out exactly, leading to the thermodynamic potential $\Omega[8]$

$$
\frac{\Omega}{V}=\beta^{-1} \sum_{n=-\infty}^{\infty} \int \frac{d^{d} k}{(2 \pi)^{d}} \ln \left(-i \hbar \omega_{n}+\frac{\hbar^{2} k^{2}}{2 m}-\mu\right)
$$

apart from irrelevant constant terms independent of $\beta$ and $\mu$. Here, $m$ and $\lambda$ are the mass and the de Broglie thermal wavelength $\lambda=\sqrt{2 \pi \hbar^{2} \beta / m}$ of the particles, and $\mu<0$ is the chemical potential. The argument of the $\log$ arithm is recognized as the kernel of the time-dependent Schrödinger equation in the imaginary-time formalism, describing a particle of mass $m$ at finite temperature in a constant background potential $-\mu$. As indicated by the single set of frequency sum and momentum integrals, Eq. (10) is a one-loop result that, because the system is noninteracting, is exact. The momentum integral is best carried out by using the Schwinger propertime representation of the logarithm and Poisson's summation formula

$$
\sum_{n=-\infty}^{\infty} e^{2 \pi i n a}=\sum_{w=-\infty}^{\infty} \delta(a-w)
$$

to replace the summation over $n$ by one over $w$. The integrals over the Schwinger propertime parameter and the momentum variable are then easily carried out to yield the familiar fugacity series [8] 


$$
\frac{\Omega}{V}=-\frac{1}{\beta \lambda^{d}} \sum_{w=1}^{\infty} w^{-\tau} e^{-\alpha w}
$$

where $\tau=d / 2+1$ and $\alpha=-\beta \mu>0$. In Eq. (12), the singular zero-temperature contribution corresponding to $w=0$ is omitted. A more careful derivation of the thermodynamic potential (10) reveals the presence of an extra convergence factor $\exp \left(i \omega_{n} \eta\right)$ typical for nonrelativistic theories, where $\eta$ is a small positive parameter that is taken to zero only after the frequency sum is evaluated [6]. This convergence factor suppresses the $T=0$ contribution (see below). Physically, the series (12) denotes the sum over closed worldlines that wind $w$ times around the imaginary time axis [10]. The first factor in the summand measures the configurational entropy of the loops, while the second, with $\alpha$ being proportional to the worldline tension, is a Boltzmann factor, weighing loops according to their length measured by $w$. Loops with $w>1$ correspond to exchange rings of $w$ particles which are cyclically permuted after an imaginary time $\hbar \beta$, as they appear in Feynman's theory of the lambda transition in superfluid ${ }^{4} \mathrm{He}[11]$.

The expression (10) can alternatively be evaluated by keeping the momentum integral to the end and instead of the Poisson formula using the identity (4). For nonrelativistic free theories, the summand there includes the convergence factor $\exp \left(i \omega_{n} \eta\right)$ that suppresses the $T=0$ part [6]. The thermodynamic potential then takes the well-known form [8]

$$
\frac{\Omega}{V}=-\frac{1}{\beta \lambda^{d}} F_{d / 2+1}(\alpha)
$$

where

$$
F_{\tau}(\alpha)=\frac{2}{\Gamma(\tau)} \int_{0}^{\infty} d q \frac{q^{2 \tau-1}}{e^{q^{2}+\alpha}-1}
$$

with $q$ the dimensionless loop variable defined by $q^{2}=$ $\beta \hbar^{2} k^{2} / 2 m$.

On comparing the two alternative evaluations of the thermodynamic potential it follows that

$$
\sum_{w=1}^{\infty} w^{-\tau} \mathrm{e}^{-\alpha w}=\frac{2}{\Gamma(\tau)} \int_{0}^{\infty} d q \frac{q^{2 \tau-1}}{e^{q^{2}+\alpha}-1}
$$

where in the first representation the momentum integral has been carried out, while in the second it still has to be performed. The equivalence of both results is easily established by expanding the integrand in a geometric series and using the integral representation of the Gamma function.

\section{B. High-Temperature Expansion}

Next, the function $F_{\tau}(\alpha)$ is expanded in a hightemperature series. As mentioned in the Introduction, such an expansion is an important computational tool for interacting theories, where exact results are absent. The standard approach is to use the identity (4) backwards, i.e., the Laurent expansion (8) of the Bose-Einstein distribution function. The zero-frequency mode, corresponding to the first term in the Laurent expansion, gives the contribution

$$
F_{\tau}^{0}(\alpha)=\frac{2}{\Gamma(\tau)} \int_{0}^{\infty} d q \frac{q^{2 \tau-1}}{q^{2}+\alpha}=\alpha^{\tau-1} \Gamma(1-\tau)
$$

which for $d=3$ leads to a nonanalytic contribution of the form $\sim \alpha^{3 / 2}$.

As an aside, this contribution due to the zerofrequency mode can also be obtained from the worldline loop representation by replacing the sum over $w$ in $F_{\tau}(\alpha)$ by an integral:

$$
\begin{aligned}
& \sum_{w=1}^{\infty} w^{-\tau} e^{-\alpha w} \rightarrow \int_{0}^{\infty} d w w^{-\tau} e^{-\alpha w} \\
& =\alpha^{\tau-1} \Gamma(1-\tau)=F_{\tau}^{0}(\alpha)
\end{aligned}
$$

The zero-frequency mode $\phi_{0}(\mathbf{k})$ is important for our purposes as it determines the critical properties of the free Bose gas. To illustrate this, we differentiate the zerofrequency mode contribution twice with respect to the chemical potential $\mu$ to obtain the known result that the compressibility $\kappa$ of an ideal Bose gas diverges as $\kappa \sim(-\mu)^{\tau-3}$ when the condensation temperature $T_{0}$ is approached from above, where $\mu$ vanishes as $\mu \sim$ $-\left(T-T_{0}\right)^{2 /(d-2)}$. For $d=3$, this implies that

$$
\kappa \propto(-\mu)^{-1 / 2} \sim\left(T-T_{0}\right)^{-1}
$$

and gives as value for the specific-heat critical exponent $\alpha_{c e}=-1$ [12]. Describing a free theory, this one-loop result is exact.

Despite being noninteracting, a free Bose gas is not in the universality class of the Gaussian model, but in that of the spherical model [12]. The nontrival exponents derive from the constraint that the total number of particles be fixed. Without this constraint, the chemical potential would vanish instead as $\mu \sim T_{0}-T$ irrespective of the dimension. This would result in Gaussian exponents describing $\mathrm{BEC}$ in a free Bose gas at constant pressure [12]. In four space dimensions, corresponding to the upper critical dimension, and above (i. e., $d \geq 4$ ), the exponents of the spherical and Gaussian model coincide.

As mentioned before, the zero-temperature contribution vanishes because of the presence of a convergence factor in nonrelativistic free theories. More in line with the present approach, it vanishes even without including this factor,

$$
-F_{\tau}^{\infty}(\alpha)=\frac{1}{\Gamma(\tau)} \int_{0}^{\infty} d q q^{2 \tau-1}=0
$$


because integrals over polynomials give zero within the dimensional regularization scheme [13].

The rest of the terms in the high-temperature expansion, corresponding to the last terms in the Laurent expansion (8), are analytic. These contributions are obtained in the standard approach by expanding the summand in a high-temperature Taylor series before carrying out the momentum integral and the frequency sum, with each term giving a Riemann zeta function

$$
\zeta(s)=\sum_{m=1}^{\infty} \frac{1}{m^{s}}
$$

Specifically, one finds [14]

$$
F_{\tau}^{\beta}(\alpha)=\sum_{l=0}^{\infty} \frac{\zeta(\tau-l)}{\Gamma(l+1)}(-\alpha)^{l}
$$

with $\alpha$ the dimensionless expansion parameter. The condensation temperature is determined by these contributions due to the gapped thermal modes. At that temperature, the chemical potential vanishes, so that the equation for the particle number density $n$,

$$
n=-\frac{1}{V} \frac{\partial \Omega}{\partial \mu}
$$

with $F_{\tau}(\alpha)$ in Eq. (13) replaced with $F_{\tau}^{\beta}(\alpha)$, gives the condensation temperature

$$
k_{\mathrm{B}} T_{0}=\frac{2 \pi \hbar^{2}}{m}\left(\frac{n}{\zeta(d / 2)}\right)^{2 / d}
$$

In other words, although the critical exponents of BEC in a free Bose gas are determined entirely by the static, zero-frequency mode, the condensation temperature, being nonuniversal, is determined entirely by the dynamic, gapped thermal modes.

It is natural to ask whether the series (21) can also be extracted directly from the integral representation given in Eq. (14), where the frequency sum has already been carried out, without returning to a representation involving such a sum. To show that the answer is affirmative, we expand the integrand there in a high-temperature series. Each term thus generated contains an integral of the form

$$
\int_{0}^{\infty} \frac{d q}{q} \frac{q^{2 t}}{e^{p q^{2}}-1}
$$

and derivatives thereof with respect to the parameter $p$ (which is set to unity at the end). For $t \leq 1$, these loop integrals diverge in the infrared. We handle the divergences using zeta function regularization, by analytically continuing the following equation

$$
2 \int_{0}^{\infty} \frac{d q}{q} \frac{q^{2 t}}{e^{p q^{2}}-1}=\Gamma(t) \zeta(t) p^{-t}
$$

with $t$ initially chosen large enough so that the integral converges, to arbitrary values of $t$. With this regularization scheme, the high-temperature expansion (21) easily follows,

$$
\begin{aligned}
& \frac{2}{\Gamma(\tau)} \int_{0}^{\infty} d q \frac{q^{2 \tau-1}}{e^{q^{2}+\alpha}-1} \stackrel{\text { Taylor }}{\longrightarrow} \sum_{l=0}^{\infty} \frac{\zeta(\tau-l)}{\Gamma(l+1)}(-\alpha)^{l} \\
& =F_{\tau}^{\beta}(\alpha),
\end{aligned}
$$

showing that the contributions due to the gapped thermal modes can indeed (when properly regularized) be extracted directly from the function $F_{\tau}(\alpha)$ in Eq. (14) without the need to return to the representation involving a frequency sum. This result is quite general and will in the next subsection be illustrated for a relativistic system.

\section{Relativistic Free Bose Gas}

To show that the conclusions of the previous section by no means hinge on the form of the nonrelativistic spectrum, leading to the specific structure of the integrand in Eq. (14), the same analysis for a relativistic free Bose gas is given in this section. The system is considered at zero chemical potential $\mu=0$. It has no phase transition then, but the algebra is simpler than with a chemical potential included [15], without losing any of the generalities we wish to point out.

The analog of expression (10) for the nonrelativistic Bose gas reads [16]

$$
\frac{\Omega}{V}=\frac{1}{2 \beta} \sum_{n=-\infty}^{\infty} \int \frac{d^{d} k}{(2 \pi)^{d}} \ln \left(\hbar^{2} \omega_{n}^{2}+\hbar^{2} c^{2} k^{2}+m^{2} c^{4}\right)
$$

with $c$ the speed of light. The argument of the logarithm is now recognized as the kernel of the Klein-Gordon equation in the imaginary-time formalism, describing a relativistic free scalar particle of mass $m$ at finite temperature. Being a noninteracting system, the one-loop result (27) is exact. With the help of the identity (4), the thermodynamic potential becomes 


$$
\begin{aligned}
\frac{\Omega}{V} & =\int \frac{d^{d} k}{(2 \pi)^{d}}\left[\frac{1}{2} \sqrt{\hbar^{2} c^{2} k^{2}+m^{2} c^{4}}+\beta^{-1} \ln \left(1-e^{-\beta \sqrt{\hbar^{2} c^{2} k^{2}+m^{2} c^{4}}}\right)\right] \\
& =\frac{(\hbar c)^{-d} \beta^{-(d+1)}}{(4 \pi)^{d / 2}}\left[\frac{1}{\Gamma(d / 2)} \int_{0}^{\infty} d q q^{d-1} \sqrt{q^{2}+\left(\beta m c^{2}\right)^{2}}-\frac{\Gamma(2 \tau)}{\Gamma(\tau)} H_{\tau}\left(\beta m c^{2}\right)\right],
\end{aligned}
$$

where the new integration variable is $q=\beta \hbar c k$ and the thermal part of the thermodynamic potential is determined by the function

$$
H_{\tau}(y)=\frac{1}{\Gamma(2 \tau)} \int_{0}^{\infty} \frac{d q}{\sqrt{q^{2}+y^{2}}} \frac{q^{2 \tau-1}}{e^{\sqrt{q^{2}+y^{2}}}-1}
$$

with $\tau=d / 2+1$. This function [16] is the relativistic analog of the function $F_{\tau}(\alpha)$ in Eq. (14).

The high-temperature expansion of the function $H_{\tau}(y)$ in $d=3$ was computed by Dolan and Jackiw [7]. To deal with logarithmic divergences that arise because four spacetime dimensions corresponds to the upper critical dimension, dimensional regularization was used by considering $d=3-\epsilon$, with $\epsilon$ taken to zero at the end. For convenience, $\tau=5 / 2-\epsilon / 2$ was only used in the integrand, whereas $\epsilon$ was set to zero from the start in the prefactor in Eq. (29). These authors used Eq. (5) backwards, i.e., Eq. (8), expanded the summand in a Taylor series, integrated term by term, and finally carried out the remaining frequency sum in each term, with the result $[7]$ :

$$
\begin{aligned}
H_{5 / 2}(y) & =\frac{\pi^{4}}{360}-\frac{\pi^{2}}{96} y^{2}+\frac{\pi}{48} y^{3} \\
& +\frac{1}{128}\left[\ln \left(\frac{y}{4 \pi}\right)+\gamma-\frac{3}{4}\right] y^{4}+\mathcal{O}\left(y^{6}\right)
\end{aligned}
$$

where $\gamma$ is Euler's constant and $y=\beta m c^{2}$ the dimensionless expansion parameter. The nonanalytic cubic contribution is due to the zero-frequency mode represented by the first term in the Laurent expansion of the Bose-
Einstein distribution function in Eq. (29):

$$
H_{5 / 2}^{0}(y)=\frac{1}{\Gamma(5)} \int_{0}^{\infty} d q \frac{q^{4}}{q^{2}+y^{2}}=\frac{\pi}{48} y^{3},
$$

where analytic regularization is used to handle the ultraviolet divergence. The second term in the Laurent expansion of the Bose-Einstein distribution function gives

$$
\begin{aligned}
-H_{5 / 2}^{\infty}(y) & =-\frac{1}{2} \frac{1}{\Gamma(5)} \int_{0}^{\infty} d q \frac{q^{4-\epsilon}}{\sqrt{q^{2}+y^{2}}} \\
& =\frac{1}{128}\left[-\frac{1}{\epsilon}+\ln \left(\frac{y}{2}\right)+\frac{7}{12}\right] y^{4},
\end{aligned}
$$

while the gapped thermal modes yield

$$
\begin{aligned}
& H_{5 / 2}^{\beta}(y)=\frac{\pi^{4}}{360}-\frac{\pi^{2}}{96} y^{2} \\
& +\frac{1}{128}\left[\frac{1}{\epsilon}-\ln (2 \pi)+\gamma-\frac{4}{3}\right] y^{4}+\mathcal{O}\left(y^{6}\right),
\end{aligned}
$$

so that $H_{5 / 2}(y)=H_{5 / 2}^{0}(y)-H_{5 / 2}^{\infty}(y)+H_{5 / 2}^{\beta}(y)$. An extra minus sign is included in the left hand of Eq. (32), so that $H_{5 / 2}^{0}$ is the $T=0$ contribution that has to be subtracted from $H_{\tau}^{\beta}(\alpha)$ to arrive at the purely thermal contributions $H_{\tau}(\alpha)$.

We next rederive this result using the algorithm given above and extract it directly from the integral representation (29), by expanding the integrand in a Taylor series. Applying zeta function regularization to handle the infrared divergences as before [see Eq. (25)], we obtain

$$
\begin{aligned}
\frac{1}{\Gamma(5)} \int_{0}^{\infty} \frac{d q}{\sqrt{q^{2}+y^{2}}} \frac{q^{4-\epsilon}}{e^{\sqrt{q^{2}+y^{2}}}-1} \stackrel{\text { Taylor }}{\longrightarrow} & \frac{\pi^{4}}{360}-\frac{\pi^{2}}{96} y^{2}+\frac{1}{128}\left[\frac{1}{\epsilon}+2 \zeta^{\prime}(0)+\gamma-\frac{4}{3}\right] y^{4} \\
& +\frac{\zeta^{\prime}(-2)}{768} y^{6}+\frac{\zeta^{\prime}(-4)}{24576} y^{8}+\frac{\zeta^{\prime}(-6)}{1474560} y^{10}+\mathcal{O}\left(y^{12}\right),
\end{aligned}
$$


where this series is carried out to higher order. To show that this expression coincides with $H_{5 / 2}^{\beta}(y)$, we differentiate the identity [17]

$$
\zeta(s)=2^{s} \pi^{s-1} \sin \left(\frac{1}{2} \pi s\right) \Gamma(1-s) \zeta(1-s),
$$

to obtain for $s$ a (positive or negative) even integer:

$$
\zeta^{\prime}(s)=2^{s-1} \pi^{s} \cos \left(\frac{1}{2} \pi s\right) \Gamma(1-s) \zeta(1-s)
$$

or specifically

$$
\begin{aligned}
& \zeta^{\prime}(0)=-\frac{1}{2} \ln (2 \pi), \quad \zeta^{\prime}(-2)=-\frac{1}{4 \pi^{2}} \zeta(3), \\
& \zeta^{\prime}(-4)=\frac{3}{4 \pi^{4}} \zeta(5), \quad \zeta^{\prime}(-6)=-\frac{45}{8 \pi^{6}} \zeta(7) .
\end{aligned}
$$

It now immediately follows that Eq. (34) gives the contributions due to the gapped thermal modes, so that the results obtained in the standard manner and the one used here indeed coincide.

Given that also the zero-frequency mode contribution as well as the $T=0$ part can be extracted from the integral representation (29), by identifying them with the first two terms in the Laurent expansion of the BoseEinstein distribution function, it follows that no backtracking to a representation involving a frequency sum is required, provided zeta function regularization is applied. Moreover, the simple, yet powerful algorithm discussed here is easily implemented using an algebraic manipulation program to generate large orders in a hightemperature expansion for a wide class of problems, including fermionic systems (with the obvious changes), as will now be demonstrated.

\section{FERMIONIC INTEGRALS}

\section{A. Free Fermi Gas}

In this subsection, a nonrelativistic free, spinless Fermi gas is considered. The thermodynamic potential is given by the one-loop expression (10) for a Bose gas with an extra minus sign included to account for the antisymmetry of the fermionic field. Using Poisson's summation formula (11), the thermodynamic potential is readily written as a fugacity series [8]

$$
\frac{\Omega}{V}=-\frac{1}{\beta \lambda^{d}} \sum_{w=1}^{\infty}(-1)^{w+1} w^{-\tau} e^{-\alpha w},
$$

where $\tau=d / 2+1$ and $\alpha=-\beta \mu>0$ again. As for a Bose gas, this series can be understood as representing a sum over closed worldlines winding around the imaginary time axis $w$ times, with each winding accompanied by a factor $(-1)$ typical for fermions.
Alternatively, the thermodynamic potential can be evaluated using the identity [6]

$$
\frac{1}{\beta} \sum_{n=-\infty}^{\infty} \frac{1}{-i \hbar \omega_{n}+z}=\frac{1}{2}-f(z)
$$

where $\omega_{n}=\pi \beta^{-1}(2 n+1)$ are the fermionic Matsubara frequencies and $f(z)$ is the Fermi-Dirac distribution function

$$
f(z)=\frac{1}{e^{\beta z}+1}=\frac{1}{2}+\mathcal{O}(\beta z)
$$

The first term at the right hand of the identity (39) gives the result for zero temperature, while the second gives the thermal contribution. Nonrelativistic free theories include again a convergence factor which suppresses the $T=0$ part. The thermodynamic potential then takes the same form (13) as for a Bose gas with the function $F_{\tau}(\alpha)$ replaced with the function

$$
G_{\tau}(\alpha)=\frac{2}{\Gamma(\tau)} \int_{0}^{\infty} d q \frac{q^{2 \tau-1}}{e^{q^{2}+\alpha}+1}
$$

The equivalence of the two representations, implying

$$
\sum_{w=1}^{\infty}(-1)^{w+1} w^{-\tau} e^{-\alpha w}=\frac{2}{\Gamma(\tau)} \int_{0}^{\infty} d q \frac{q^{2 \tau-1}}{e^{q^{2}+\alpha}+1}
$$

is easily established.

\section{B. High-Temperature Expansion}

As for a bosonic system, the standard hightemperature series expansion of the function $G_{\tau}(\alpha)$ is to use the identity (39) backwards and to replace the Fermi-Dirac distribution function again with an expression involving a frequency sum:

$$
f(z)=\frac{1}{2}-\frac{2}{\beta} \sum_{n=0}^{\infty} \frac{z}{\hbar^{2} \omega_{n}^{2}+z^{2}}
$$

where the first term at the right hand subtracts the $T=0$ part encoded in the sum. Unlike bosonic systems, fermionic systems do not have a zero-frequency mode as none of the Matsubara frequencies (1) vanishes.

Following the algorithm advocated here, we instead derive this expansion directly from the integral representation of the function $G_{\tau}(\alpha)$ given in Eq. (41) without first returning to an expression involving a frequency sum. Expanding the integrand in Eq. (41) in a Taylor series, we encounter loop integrals of the form

$$
\int_{0}^{\infty} \frac{d q}{q} \frac{q^{2 t}}{e^{p q^{2}}+1}
$$


which for $t \leq 0$ are infrared divergent. These divergences are handled again using zeta function regularization, where the following equation

$$
2 \int_{0}^{\infty} \frac{d q}{q} \frac{q^{2 t}}{e^{p q^{2}}+1}=\Gamma(t)\left(1-2^{1-t}\right) \zeta(t) p^{-t}
$$

with $t$ initially chosen large enough so that the integral converges, is analytically continued to arbitrary values of $t$. The high-temperature expansion now follows as:

$$
\begin{aligned}
& \frac{2}{\Gamma(\tau)} \int_{0}^{\infty} d q \frac{q^{2 \tau-1}}{e^{q^{2}+\alpha}+1} \\
& \stackrel{\text { Taylor }}{\longrightarrow} \sum_{l=0}^{\infty}\left(1-2^{1-\tau+l}\right) \frac{\zeta(\tau-l)}{\Gamma(l+1)}(-\alpha)^{l}=G_{\tau}^{\beta}(\alpha) .
\end{aligned}
$$

This result can be checked by substituting Eq. (42) and differentiating the resulting equation arbitrary many times with respect to the dimensionless expansion pa- rameter $\alpha$ and setting that parameter to zero at the end, leading to

$$
\sum_{w=1}^{\infty}(-1)^{w+1} w^{-t}=\left(1-2^{1-t}\right) \zeta(t)
$$

which is an identity.

\section{Relativistic Free Fermi Gas}

As last noninteracting example, a relativistic free Fermi gas at zero chemical potential is considered, consisting of particles (but no antiparticles, which would lead to an extra degeneracy factor) with half-integer spin $\sigma$. The thermodynamic potential is given by the expression (27) for the Bose gas with an extra spin multiplication factor and a minus sign, i. e., $-(2 \sigma+1)$, included. With $\omega_{n}$ now denoting fermionic Matsubara frequencies, the one-loop expression is readily cast in the form

$$
\frac{\Omega}{V}=-(2 \sigma+1) \frac{(\hbar c)^{-d} \beta^{-(d+1)}}{(4 \pi)^{d / 2}}\left[\frac{1}{\Gamma(d / 2)} \int_{0}^{\infty} d q q^{d-1} \sqrt{q^{2}+\left(\beta m c^{2}\right)^{2}}-\frac{\Gamma(2 \tau)}{\Gamma(\tau)} I_{\tau}\left(\beta m c^{2}\right)\right],
$$

where the thermal part is determined by the function

$$
I_{\tau}(y)=\frac{1}{\Gamma(2 \tau)} \int_{0}^{\infty} \frac{d q}{\sqrt{q^{2}+y^{2}}} \frac{q^{2 \tau-1}}{e^{\sqrt{q^{2}+y^{2}}}+1} .
$$

For $\tau=5 / 2$, it has the high-temperature expansion [7]

$$
\begin{aligned}
& I_{5 / 2}(y)=\frac{7 \pi^{4}}{2880}-\frac{\pi^{2}}{192} y^{2} \\
& -\frac{1}{128}\left[\ln \left(\frac{y}{\pi}\right)+\gamma-\frac{3}{4}\right] y^{4}+\mathcal{O}\left(y^{6}\right),
\end{aligned}
$$

where because of the absence of a zero-frequency mode, all terms are analytic in the dimensionless expansion pa- rameter $y=\beta m c^{2}$.

Apart from a minus sign, the $T=0$ contribution is the same as that for a Bose gas given in Eq. (32):

$$
\begin{aligned}
& -I_{5 / 2}^{\infty}(y)=\frac{1}{2} \frac{1}{\Gamma(5)} \int_{0}^{\infty} d q \frac{q^{4-\epsilon}}{\sqrt{q^{2}+y^{2}}} \\
& =\frac{1}{128}\left[\frac{1}{\epsilon}-\ln \left(\frac{y}{2}\right)-\frac{7}{12}\right] y^{4},
\end{aligned}
$$

where again an extra minus sign is included in the left hand of this definition, so that $I_{5 / 2}^{0}$ gives the $T=0$ contribution (and not its negative).

Using the algorithm discussed here, we expand the integrand of Eq. (49) in a Taylor series to obtain as contribution due to the gapped thermal modes:

$$
\begin{aligned}
& \frac{1}{\Gamma(2 \tau)} \int_{0}^{\infty} \frac{d q}{\sqrt{q^{2}+y^{2}}} \frac{q^{2 \tau-1}}{e^{\sqrt{q^{2}+y^{2}}+1} \stackrel{\text { Taylor }}{\longrightarrow}} \frac{7 \pi^{4}}{2880}-\frac{\pi^{2}}{192} y^{2}+\frac{1}{128}\left[-\frac{1}{\epsilon}-2 \zeta^{\prime}(0)-\gamma-2 \ln (2)+\frac{4}{3}\right] y^{4} \\
&-\frac{7}{768} \zeta^{\prime}(-2) y^{6}-\frac{31}{24576} \zeta^{\prime}(-4) y^{8}-\frac{127}{1474560} \zeta^{\prime}(-6) y^{10}+\mathcal{O}\left(y^{12}\right)
\end{aligned}
$$


which, with the help of the identities (37) and together with $I_{5 / 2}^{\infty}(y)$, reproduces the result $(50)$ (expanded to higher order).

These last two examples illustrate that the hightemperature expansion we use in conjunction with zeta function regularization of infrared divergences is also valid for fermionic systems.

\section{High-Temperature Expansion of BCS Theory}

In Ref. [3], the BCS theory was considered at finite temperature using the approach advocated here. This example is included to point out a shift in approach when going from a noninteracting to an interacting system. The main difference with a noninteracting system is of course that one-loop results no longer are exact, meaning that not all the degrees of freedom can be integrated out in an exact manner to obtain the thermodynamic potential as a known function of the thermodynamic parameters $\alpha$ and $\beta$. Instead, irrelevant degrees of freedom are integrated out (usually only perturbatively) to arrive at an effective theory of the Landau form expressed in terms of an order parameter describing the phase transition. The order parameter, whose value is zero in the symmetric, disordered state and nonzero in the ordered state with spontaneously broken symmetry, determines the critical properties of the system close to the phase transition, where it disappears.

After linearizing the quartic interaction of the BCS theory by a suitable Hubbard-Stratonovich transformation, the fermionic degrees of freedom can be integrated out exactly in a one-loop calculation to yield for a uniform BCS superconductor:

$$
\begin{aligned}
\frac{\Omega_{1}(\Delta)}{V} & =-2 \int \frac{d^{3} k}{(2 \pi)^{3}}\left[\frac{1}{2} E(k)\right. \\
& \left.+\beta^{-1} \ln \left(1+e^{-\beta E(k)}\right)\right] .
\end{aligned}
$$

Here, the frequency sum has been carried out and

$$
E(k)=\sqrt{\xi^{2}(k)+|\Delta|^{2}},
$$

is the BCS spectrum with the order parameter $\Delta$ providing an energy gap at the Fermi surface. This spectrum replaces the excitation spectrum $\xi(k)=\hbar^{2} k^{2} / 2 m-\mu$ of the elementary fermionic excitations of mass $m$ in the normal state, where the energy is measured relative to the chemical potential $\mu$, which, contrary to a dilute free Fermi gas, is positive in the weak-coupling BCS limit and given by the Fermi energy $\mu=\hbar^{2} k_{\mathrm{F}}^{2} / 2 m$, with $\hbar k_{\mathrm{F}}$ the Fermi momentum. The prefactor 2 in Eq. (53) arises because the fermions come in two species, with spin up and down. The true grand canonical potential $\Omega$, depending only on the thermodynamic parameters $\alpha$ and $\beta$, is obtained from $\Omega(\Delta)$ by integrating out $\Delta$. This can only be done perturbatively, with the simplest approximation corresponding to the saddle point of the integral, where the order parameter is considered to be a nonfluctuating field.

To the one-loop expression (53), the tree contribution is to be added

$$
\frac{\Omega_{0}(\Delta)}{V}=-\frac{|\Delta|^{2}}{g_{\mathrm{B}}},
$$

where $g_{\mathrm{B}}<0$ is the (bare) coupling constant of the local BCS interaction term, representing the effective attraction between fermions. The pure quantum zerotemperature term in Eq. (53) gives, among other contributions, a quadratic term which can be combined with the tree contributions to yield the renormalized coupling constant $g$,

$$
\frac{1}{g}=\frac{1}{g_{\mathrm{B}}}+\frac{1}{2} \int \frac{d^{3} k}{(2 \pi)^{3}} \frac{1}{|\xi(k)|} .
$$

The integral diverges in the ultraviolet, to regularize it we introduce a momentum cutoff $\hbar \Lambda$ to obtain

$$
\frac{1}{g}=\frac{1}{g_{\mathrm{B}}}+\frac{m}{2 \pi^{2} \hbar^{2}} \Lambda+\mathcal{O}\left(\Lambda^{0}\right)
$$

where the irrelevant finite part of the integral is omitted. The weak-coupling BCS limit corresponds to taking $g_{\mathrm{B}} \rightarrow 0$ from below.

With the loop integration approximated by

$$
\int \frac{d^{3} k}{(2 \pi)^{3}} \rightarrow \nu(0) \int_{-\infty}^{\infty} d \xi
$$

where $\nu(0)=m k_{\mathrm{F}} / 2 \pi^{2} \hbar^{2}$ is the density of states per spin degree of freedom at the Fermi level, the thermal part of the thermodynamic potential (53) becomes of a form encountered in a relativistic free Fermi gas [7], with the dimensionless integration variable $q=\beta \xi$. The approximation consists of extending the range of the $\xi$ integration from $-\mu \leq \xi<\infty$ to $-\infty<\xi<\infty$. The problem thus reduces to one in a single space dimension $(d=1)$, corresponding to the value $\tau=3 / 2$. To handle logarithmic divergences that arise, we again dimensional regularize the integral and consider the problem in $(1-\epsilon)$ dimensions instead. We proceed in the same way as before and rather than returning to an expression involving a frequency sum by using Eq. (39) backwards, we expand the integrand in Eq. (29) in a high-temperature series and apply zeta function regularization to handle infrared divergences. This gives as contributions due to the gapped thermal modes: [3] 


$$
\int \frac{d^{3} k}{(2 \pi)^{3}} \ln \left(1+e^{-\beta \sqrt{\xi^{2}(k)+|\Delta|^{2}}}\right)=\beta^{-1} \nu(0)\left\{\frac{\pi^{2}}{6}+\frac{1}{2}\left[\frac{1}{\epsilon}+2 \zeta^{\prime}(0)+\gamma+2 \ln (2)\right] y^{2}+\frac{7}{8} \zeta^{\prime}(-2) y^{4}+\mathcal{O}\left(y^{6}\right)\right\}
$$

Because the order parameter appears only in the dimensionless expansion parameter $y=\beta|\Delta|$, the hightemperature expansion is tantamount to a Landau expansion in $|\Delta|$. Contact with the standard approach is established through the identities (37) and the substitution

$$
\frac{1}{\epsilon} \rightarrow \ln \left(\beta \hbar \omega_{\mathrm{D}}\right)
$$

where the Debye energy $\hbar \omega_{\mathrm{D}}$, being a measure of the inverse lattice spacing, is the physical ultraviolet cutoff and the temperature $\beta^{-1}$ is the relevant infrared scale. This correspondence between the pole $1 / \epsilon$ of dimensional regularization and the logarithm $\ln (\Lambda)$ appearing in momentum cutoff regularization is commonly used in the context of quantum field theory, and can be verified explicitly here by working with the Debye energy as a cutoff from the beginning instead of using dimensional regularization. Adding the tree and the one-loop contributions, we then find

$$
\frac{\Omega_{0}(\Delta)+\Omega_{1}(\Delta)}{V}=-\beta^{-2} \nu(0)\left\{\frac{\pi^{2}}{3}+\left[\ln \left(\beta \hbar \omega_{\mathrm{D}}\right)+\gamma-\ln (\pi / 2)+\frac{1}{\nu(0) g}\right]|\beta \Delta|^{2}-\frac{7 \pi^{2}}{16} \zeta(3)|\beta \Delta|^{4}\right\}
$$

featuring the renormalized coupling constant $g$. Being a static field, the order parameter is the zero-frequency mode of the problem at hand. The critical temperature $T_{C}$ is determined by the condition that the coefficient of the quadratic term changes sign, yielding the standard result [24]

$$
k_{\mathrm{B}} T_{\mathrm{c}}=\frac{2}{\pi} e^{\gamma} \hbar \omega_{\mathrm{D}} e^{1 / \nu(0) g}
$$

with $g<0$. Using this expression for the critical temperature, we can put Eq. (61) in the canonical form [18]

$$
\begin{aligned}
\frac{\Omega_{0}(\Delta)+\Omega_{1}(\Delta)}{V} & =-\beta^{-2} \nu(0)\left[\frac{\pi^{2}}{3}-\ln \left(\frac{\beta_{c}}{\beta}\right)|\beta \Delta|^{2}\right. \\
& \left.-\frac{7 \pi^{2}}{16} \zeta(3)|\beta \Delta|^{4}\right]
\end{aligned}
$$

valid close to the critical temperature.

This illustrates first of all that our method of computing the contributions due to the gapped thermal modes in a high-temperature series expansion in conjunction with zeta function regularization of infrared divergences is also valid for an interacting system. In addition, it highlights the general approach to critical phenomena, which is to integrate out the irrelevant degrees of freedom (which include the gapped thermal and thus all fermionic modes) to determine the coefficients of the effective theory expressed in terms of the order parameter. Although irrelevant for the critical behaviour itself, the gapped thermal modes can influence the value of the critical tempera- ture through their contributions to the coefficient of the quadratic term. The critical temperature (62), which is a one-loop result, is exclusively determined by the highenergy dynamic modes as none of the Matsubara frequencies of the fermionic fields that have been integrated out vanishes. Unlike critical exponents, the critical temperature is not universal and can depend on the details of these high-energy gapped modes.

\section{CONDENSATION TEMPERATURE OF WEAKLY INTERACTING BOSE GAS}

\section{A. Effective Theory}

We next consider the condensation temperature of a uniform, weakly interacting Bose gas. Specifically, we are interested in how fluctuations in the irrelevant degrees of freedom influence this temperature. To determine this, we need, according to the effective field program (see the discussion in the preceding section), to calculate the coefficients of the effective theory expressed in terms of the order parameter by integrating out the irrelevant degrees of freedom. For BEC the irrelevant degrees of freedom are the gapped thermal modes, whose contributions are conveniently computed using the algorithm advocated here. The order parameter $\Phi$ of BEC in a weakly interacting Bose gas, introduced in Eq. (9), denoting the expectation value of the quantum field $\phi$ describing the bosons, is the zero-frequency mode. A nonzero $\Phi$ implies the presence of a condensate, with $|\Phi|^{2}$ giving the average number density $n_{\text {vac }}$ of condensed particles. This effective field program is the field-theoretic generalization of the effective classical potential introduced by Feynman 
and Kleinert [19] in their path-integral study of thermal fluctuations via a final integral over the zero-frequency component of the path variable. (See the textbook [20] for extensive applications.) Here we are left with a final functional integral over the purely space-dependent field $\Phi(\mathbf{x})$.

Whereas the condensation temperature of a free Bose gas is determined from within the normal, i.e., the hightemperature state, we determine $T_{c}$ for the weakly interacting gas instead from within the low-temperature state with a nonzero condensate $|\Phi|$.

The one-loop contribution to the thermodynamic potential of a weakly interacting uniform Bose gas is given by $[3]$

$$
\begin{aligned}
\frac{\Omega_{1}(\Phi)}{V} & =\int \frac{d^{3} k}{(2 \pi)^{3}}\left[\frac{1}{2} E(k)\right. \\
& \left.+\beta^{-1} \ln \left(1-e^{-\beta E(k)}\right)\right],
\end{aligned}
$$

where $E(k)$ is the spectrum of the elementary excitation in the superfluid state expressed in terms of the order parameter $\Phi$

$$
E(k)=\sqrt{\left(\hbar^{2} k^{2} / 2 m-\mu_{\mathrm{B}}+4 g_{\mathrm{B}}|\Phi|^{2}\right)^{2}-4 g_{\mathrm{B}}^{2}|\Phi|^{4}},
$$

where $\mu_{\mathrm{B}}$ and $g_{\mathrm{B}}$ are the bare parameters of the theory, with $g_{\mathrm{B}}>0$ the coupling constant of the repulsive two-particle contact interaction. The true thermodynamic potential $\Omega(\alpha, \beta)$ is obtained by integrating out the remaining degree of freedom, which in a first approximation is done by evaluating the integral in the saddle point, corresponding to extremizing $\Omega(\Phi)$.

Written in the form (65), the spectrum is the bosonic analog of the fermionic BCS spectrum (54) given in terms of the superconducting order parameter $\Delta$. The spectrum (65) reduces to the $T=0$ Bogoliubov spectrum [21] when the lowest-order value $|\Phi|^{2}\left(=n_{\mathrm{vac}}\right)=\mu_{\mathrm{B}} / 2 g_{\mathrm{B}}$ for $\Phi$ is inserted. This value is obtained from minimizing the tree contribution

$$
\frac{\Omega_{0}(\Phi)}{V}=-\mu_{\mathrm{B}}|\Phi|^{2}+g_{\mathrm{B}}|\Phi|^{4}
$$

It is important to note that the chemical potential $\mu_{\mathrm{B}}=$ $2 g_{\mathrm{B}} n_{\text {vac }}$ is positive here (so that the coefficient of the quadratic term is negative, leading to a potential of a form resembling a Mexican hat), setting a weakly interacting Bose gas apart from a free Bose gas which has a nonpositive chemical potential.

The pure quantum zero-temperature term in Eq. (64) gives, among other contributions, two terms of the form contained in the bare theory (66). They can be combined to yield the renormalized parameters [22]:

$$
\begin{aligned}
& \mu=\mu_{\mathrm{B}}-\frac{1}{6 \pi^{2}} g_{\mathrm{B}} \Lambda^{3} \\
& g=g_{\mathrm{B}}-\frac{m}{\pi^{2} \hbar^{2}} g_{\mathrm{B}}^{2} \Lambda,
\end{aligned}
$$

with $\hbar \Lambda$ the momentum cutoff. In addition, it gives a nonanalytic contribution $\propto m^{3 / 2}\left(g|\Phi|^{2}\right)^{5 / 2}$ induced by the gapless Goldstone mode. This one-loop contribution, which is irrelevant for our present purposes and will subsequently be ignored, is typical for a gapless contribution in five spacetime dimensions. The effective number of spacetime dimensions is five here because in nonrelativistic quantum theories, where time derivatives appear in combination with two space derivatives, the time dimension counts double compared to the (three) space dimensions.

Since Eq. (64) is already a one-loop result, it is consistent to this order to replace the bare parameters $\mu_{\mathrm{B}}, g_{\mathrm{B}}$ there with the (one-loop) renormalized ones. The renormalized coupling constant $g$ is related to the $s$-wave scattering length $a$ via $[23,24]$

$$
g=\frac{2 \pi \hbar^{2} a}{m}
$$

The zero-temperature relation between the renormalized, physical chemical potential and the average number density $n_{\mathrm{vac}}=|\Phi|^{2}$ of condensed particles then reads [6]

$$
\mu(T=0)=2 g n_{\mathrm{vac}}=\frac{4 \pi \hbar^{2} a}{m} n,
$$

where in the last step we ignored the so-called depletion of the condensate [21] and replaced $n_{\mathrm{vac}}$ with the total particle number density, which is justified because the term is already of order $g$.

Next, the thermal part in Eq. (64) is expanded in a high-temperature series using the algorithm discussed above. Writing

$$
\begin{aligned}
& \int \frac{d^{3} k}{(2 \pi)^{3}} \ln \left(1-e^{-\beta E(k)}\right) \\
& =\frac{32 \pi}{(4 \pi)^{3 / 2}} \frac{1}{\lambda^{3}} \int_{0}^{\infty} d q q^{2} \ln \left(1-e^{-\beta E(q)}\right),
\end{aligned}
$$

with $q$ the same loop variable as used for a free Bose gas, defined by $q^{2}=\beta \hbar^{2} k^{2} / 2 m$, and

$$
\beta E(q)=\sqrt{\left(q^{2}+\alpha+4 y\right)^{2}-4 y^{2}},
$$

where $y=\beta g|\Phi|^{2}$, we arrive at [3] 


$$
\begin{aligned}
& \int_{0}^{\infty} d q q^{2} \ln \left(1-e^{-\beta E(q)}\right) \stackrel{\text { Taylor }}{\longrightarrow} \\
& \int_{0}^{\infty} d q\left[q^{2} \ln \left(1-e^{-q^{2}}\right)+\frac{q^{2}}{e^{q^{2}}-1}(\alpha+4 y)-\frac{1}{2} \frac{q^{2} e^{q^{2}}}{\left(e^{q^{2}}-1\right)^{2}}(\alpha+4 y)^{2}-\frac{1}{2} \frac{1}{e^{q^{2}-1}} 4 y^{2}+\mathcal{O}\left(\beta^{3}\right)\right] \\
& \quad=-\frac{1}{2} \Gamma\left(\frac{3}{2}\right)\left[\zeta\left(\frac{5}{2}\right)-\zeta\left(\frac{3}{2}\right)(\alpha+4 y)+\frac{1}{2} \zeta\left(\frac{1}{2}\right)(\alpha+4 y)^{2}+\zeta\left(\frac{1}{2}\right) 4 y^{2}+\mathcal{O}\left(\beta^{3}\right)\right] .
\end{aligned}
$$

Contrary to the previous cases, we now have two dimensionless expansion parameters $\alpha$ and $y$, both being proportional to $\beta$.

At two loop, we consider the class of bubble diagrams (see Fig. 4 of Ref. [3]) giving the following contributions to the thermodynamic potential due to the gapped thermal modes:

$$
\frac{\Omega_{2}(\Phi)}{V}=-\frac{g}{4}\left(3 I_{1}^{2}+3 I_{2}^{2}+2 I_{1} I_{2}\right)
$$

where $I_{1}$ is the one-loop integral

$$
I_{1}=\int \frac{d^{3} k}{(2 \pi)^{3}} \frac{1}{E(k)} \frac{\hbar^{2} k^{2} / 2 m-\mu+2 g|\Phi|^{2}}{e^{\beta E(k)}-1},
$$

and a similar equation for $I_{2}$ with $2 g$ replaced with $6 g$ in the numerator. Expanding the integrals in a hightemperature expansion, we obtain as two-loop contributions

$$
\frac{\Omega_{2}(\Phi)}{V}=\frac{2 g}{\lambda^{6}}\left[\zeta^{2}\left(\frac{3}{2}\right)-2 \zeta\left(\frac{1}{2}\right) \zeta\left(\frac{3}{2}\right)(\alpha+4 y)\right]
$$

the leading term of which was considered in Ref. [3], but not the rest.

Adding the contributions together, we arrive at

$$
\frac{\Omega_{0}(\Phi)+\Omega_{1}(\Phi)+\Omega_{2}(\Phi)}{V}=c_{0}-c_{2}|\Phi|^{2}+c_{4}|\Phi|^{4}
$$

with

$$
\begin{aligned}
c_{0} & =-\frac{1}{\beta \lambda^{3}}\left\{\zeta\left(\frac{5}{2}\right)-2 \zeta^{2}\left(\frac{3}{2}\right) \delta\right. \\
& \left.-\zeta\left(\frac{3}{2}\right) \alpha\left[1-4 \zeta\left(\frac{1}{2}\right) \delta\right]+\frac{1}{2} \zeta\left(\frac{1}{2}\right) \alpha^{2}\right\},
\end{aligned}
$$

a $\Phi$-independent term, with expansion parameter

$$
\delta=g \beta / \lambda^{3}=a / \lambda
$$

For $\delta \rightarrow 0$, Eq. (78) is analogous to the first contributions due to the gapped thermal modes of a free Bose gas given by Eq. (13) with Eq. (21), albeit with $\alpha>0$ now. Furthermore,

$$
\begin{aligned}
-\beta c_{2} & =\left[\alpha+4 \zeta\left(\frac{3}{2}\right) \delta\right]\left[1-4 \zeta\left(\frac{1}{2}\right) \delta\right], \\
c_{4} & =g\left[1-12 \zeta\left(\frac{1}{2}\right) \delta\right] .
\end{aligned}
$$

It is important to note that one cannot naively set $\Phi$ to zero in Eq. (77) [with $\Omega_{2}(\Phi)$ omitted], as was done by Baym et al. [1] to argue that the zeta function regularization of infrared divergences used to obtain that result is flawed, and hope to retrieve the correct critical behavior of the compressibility, say, of a noninteracting Bose gas. As explained in Sec. (II B), universal properties are determined exclusively by the fluctuations in the zero-frequency mode. These fluctuations are, however, not considered in this section. We are interested here in the condensation temperature - a nonuniversal quantity. As argued in the Introduction, nonuniversal quantities in principle also depend on the gapped thermal modes. It is the effect of fluctuations in these dynamic modes that is summarized by Eq. (77). More specifically, the coefficients given in Eqs. (80) express the dressing of the parameters $\mu$ and $g$ by these thermal fluctuations. The one-loop corrections, first calculated in Ref. [3], have recently been rederived in Ref. [5], apparently in ignorance of the existing literature on the subject.

The condensation temperature is determined by the vanishing of the coefficient $c_{2}$, yielding

$$
\alpha_{c}=-4 \zeta\left(\frac{3}{2}\right) \delta
$$

which gives $T_{c}$ in terms of $\mu$. Experimentally, it is more realistic to consider the system at fixed particle number density and therefore take $n$ rather than $\mu$ as independent variable. The particle number density is easily obtained from Eq. (77) at $T=T_{c}$ where $\Phi$ vanishes:

$$
\begin{aligned}
n=-\left.\frac{1}{V} \frac{\partial \Omega}{\partial \mu}\right|_{T=T_{c}} & =\frac{1}{\lambda_{c}^{3}}\left\{\zeta\left(\frac{3}{2}\right)\left[1-4 \zeta\left(\frac{1}{2}\right) \delta\right]\right. \\
& \left.-\zeta\left(\frac{1}{2}\right) \alpha_{c}\right\} .
\end{aligned}
$$

Together with Eq. (81) this reduces to the free Bose gas expression: 


$$
n=\zeta\left(\frac{3}{2}\right) / \lambda_{c}^{3},
$$

so that to this order the dynamic modes do not lead to a shift in the condensation temperature. This conclusion differs from our previous one in Ref. [3], where the twoloop contributions were not properly included [4]. The additional two-loop contributions cancel the shift due to the gapped thermal modes found in Ref. [3] at one loop.

With the expression (83) for $n$, the chemical potential at $T_{c}$ can be written as

$$
\mu_{c}=4 g n=\frac{8 \pi \hbar^{2} a}{m} n,
$$

which is nothing but the Hugenholtz-Pines relation [25] between the chemical potential and the self-energy at the condensation temperature where $\Phi$ disappears. It is identical to the zero-temperature relation (70) apart from a subtle factor of 2 that can be understood by examining the Hugenholtz-Pines relation, which is an exact result, at both temperatures. It follows from Eq. (84) that the chemical potential remains positive at the condensation temperature of a weakly interacting Bose gas. This is in contrast to a free Bose gas (obtained by letting $a \rightarrow 0$ ), where the chemical potential vanishes when the condensation temperature is approached from above. Whereas the chemical potential in a free Bose gas remains zero all the way down to zero temperature, in a weakly interacting Bose gas it decreases from $4 g n$ at $T_{c}$ to $2 g n$ at zero temperature.

To justify the high-temperature expansion used, note that to lowest nontrivial order, the coefficient $c_{2}$ is given by $-\beta c_{2}=\alpha+4 \zeta\left(\frac{3}{2}\right) \delta$. The condition $c_{2}=0$ then gives the condensation temperature in terms of $\mu$ to lowest order as [3]

$$
k_{\mathrm{B}} T_{0}=\frac{2 \pi \hbar^{2}}{m}\left(\frac{\mu}{4 g \zeta\left(\frac{3}{2}\right)}\right)^{2 / 3},
$$

which is a one-loop result. Since this temperature is large for $g$ small, the high-temperature expansion is consistent with the weak-coupling assumption of perturbation theory. Using the Hugenholtz-Pines relation (which is also satisfied at this lowest nontrivial order) to replace $\mu$ with $n$, Eq. (85) takes the standard form (23) for a noninteracting Bose gas, independent of $g$. As for a free Bose gas, this result is determined entirely by the nonzero frequency modes.

Because $\alpha_{c} \propto \delta \propto g$, the coefficients (80a) and (80b) of the effective theory are close to $T_{\mathrm{C}}$ calculated up to the order $g^{2}$. In the first coefficient (78), the term $\propto \delta^{2}$ is missing. This term, given by the leading contribution in the high-temperature expansion of the third-order bubble diagram, is independent of the chemical potential and therefore irrelevant for our purposes.

\section{B. BEC in two dimensions}

For arbitrary dimension $2<d<4$, Eqs. (81) and (83) generalize to:

$$
\alpha_{c}=-4 \zeta(d / 2) \delta, \quad n=\zeta(d / 2) / \lambda_{c}^{d}
$$

where now $\delta=g \beta / \lambda^{d}$. The limiting case, $d=2$, is special because $\zeta(d / 2)$ diverges when $d \rightarrow 2$. To investigate this limit, we dimensional regularize the last equation by considering the problem in $d=2+\epsilon$, where

$$
n=\frac{2}{\lambda_{c}^{2}} \frac{1}{\epsilon} .
$$

As in Eq. (60), the pole in dimensional regularization can be connected with the logarithm appearing in the regularization with a momentum cutoff, provided the ultraviolet momentum cutoff and the relevant infrared scale are identified. Here, they are given by the inverse range of the potential $1 / a$ and the square root of the chemical potential, respectively [26], i.e.,

$$
\begin{aligned}
\frac{1}{\epsilon} \rightarrow \ln \left(\frac{\hbar}{a \sqrt{m \mu}}\right) & =-\frac{1}{2} \ln \left(\frac{8 \pi n a^{2}}{\ln \left(1 / 8 \pi n a^{2}\right)}\right) \\
& \approx \frac{1}{2} \ln \left[\ln \left(1 / 8 \pi n a^{2}\right)\right]
\end{aligned}
$$

for $\ln \left[\ln \left(1 / 8 \pi n a^{2}\right)\right] \gg 1$. In deriving this use is made of the two-dimensional relation between the chemical potential and the particle number density at the condensation temperature,

$$
\mu_{c}=\frac{8 \pi \hbar^{2} n}{m \ln \left(1 / 8 \pi n a^{2}\right)}
$$

With this correspondence, Eq. (87) leads to the wellknown expression for the critical temperature $[27,26]$

$$
k_{\mathrm{B}} T_{\mathrm{C}}=\frac{2 \pi \hbar^{2} n}{m \ln \left[\ln \left(1 / 8 \pi n a^{2}\right)\right]} .
$$

Together with the relation $\mu_{c}=4 g n$, Eq. (89) gives for the coupling constant

$$
g=\frac{2 \pi \hbar^{2}}{m \ln \left(\hbar^{2} / m \mu a^{2}\right)} .
$$

To understand this result, recall that the time dimension counts double compared to the space dimensions in a nonrelativistic quantum theory. The quantum critical behaviour of the theory was first investigated by Uzunov [28], who showed that $d=2$ (implying a total of four effective spacetime dimensions) corresponds to the upper 
critical dimension, below which a nontrivial infrared stable fixed point exists, with $g>0$. For $d>2$, the theory has a trivial infrared stable fixed point at $g=0$. The $\log -$ arithm in Eq. (91) arises because $d=2$ separates these two different cases, with the chemical potential providing an infrared cutoff, so that a finite value for $g$ and ultimately for $T_{c}$ obtains. In other words, the finite value for the condensation temperature in two dimensions hinges on the presence of quantum fluctuations.

As an aside, we note that at zero temperature, the relation between the chemical potential and particle number density differs again a subtle factor of 2 from the relation $\mu_{c}=4 g n$ we used to derive the critical temperature (90). As in $d=3$, this factor is needed for the HugenholtzPines relation to be satisfied at both temperatures. It implies that the analog of Eq. (89) at zero temperature also differs a factor of 2 , which is indeed what is found by Fisher and Hohenberg [26].

\section{Discussion}

Path-integral Monte Carlo simulations by Grüter, Ceperley, and Laloë [29] of the microscopic model describing a weakly interacting Bose gas restricted to relatively few (up to 216) particles in three dimensions found an increase in the condensation temperature linear in the scattering length,

$$
\frac{T_{c}-T_{0}}{T_{0}}=\operatorname{Can}^{1 / 3}
$$

with $C=0.34 \pm 0.06$. Since the dynamic modes do not contribute to the shift linear in $a$, the coefficient $C$ can also be calculated in the static, classical theory. Recent Monte-Carlo simulations [30] of that static theory gave larger values: $C=1.32 \pm 0.02$ and $1.29 \pm 0.05$, respectively. An intuitive understanding of the increase in $T_{c}$ was given in Ref. [29], where it was pointed out that a moderate repulsive interaction suppresses density fluctuations. This results in a more homogeneous system and facilitates the formation of large exchange rings necessary for BEC, which then takes place already at a higher temperature than in a free Bose gas. When the repulsive interaction increases further, the exchange is obstructed because it becomes more difficult for the particles to move. This leads to a lowering of the critical temperature as seen, for example, in liquid ${ }^{4} \mathrm{He}$. A free gas with ${ }^{4} \mathrm{He}$ parameters at vapor pressure would have a condensation temperature of about $3 \mathrm{~K}$, whereas liquid ${ }^{4} \mathrm{He}$ becomes superfluid at the lower temperature of $2.17 \mathrm{~K}$.

The full thermodynamic potential of an interacting Bose system can close to $T_{c}$ be written in terms of the exchange rings by slightly generalizing Eq. (38) for a free Bose gas [31]:

$$
\frac{\Omega}{V} \propto \sum_{w=1}^{\infty} w^{-\tau} e^{-\left(\alpha-\alpha_{c}\right) w}
$$

where now the exponent $\tau$ has the general form

$$
\tau=\frac{d}{D}+1
$$

with $D$ the fractal dimension of the worldlines, which is $D=2$ for a free Bose gas. The worldline tension determined by $\alpha-\alpha_{c}$ vanishes when the condensation temperature is reached from above as

$$
\alpha-\alpha_{\mathrm{c}} \propto\left(T-T_{\mathrm{c}}\right)^{1 / \sigma}
$$

where $\sigma$ is a second exponent, which for a free Bose gas takes the value $\sigma=d / 2-1$ [see above Eq. (18)]. The two exponents $\sigma$ and $\tau$ determine all the critical exponents characterizing the phase transition through scaling relations [31]. BEC in a weakly interacting Bose gas is in the same universality class as the lambda transition of liquid ${ }^{4} \mathrm{He}$, so that the critical exponents describing BEC are accurately known. In going from a free Bose gas to strongly interacting ${ }^{4} \mathrm{He}$, the value of the fractal dimension $D$ of the worldlines changes only slightly from $D=2$ to $D \approx 1.96$, with most of the interaction effects entering $\sigma$, which changes from $\sigma=1 / 2$ to $\sigma \approx 0.76$ [31]. The value of the fractal dimension follows from the relation [31] $D=2-\eta$, with $\eta$ the Fisher exponent, determining the anomalous dimension of the order parameter $\Phi$.

For an ideal Bose gas, the value $D=2$ derives from the quadratic form of the energy spectrum $E(k)=\hbar^{2} k^{2} / 2 m$. A different value is obtained by modifying the free spectrum to $E(k) \sim k^{D}$, with $D \neq 2$. It was noticed by Gunton and Buckingham [12] that an ideal gas with $D=3 / 2$, so that $E(k) \sim k^{3 / 2}$, produces a value of the specific-heat critical exponent $\alpha_{c e}=0$ close to the experimental value $\alpha_{\text {ce }} \approx-0.01$ for ${ }^{4} \mathrm{He}$ [32]. However, this is a somewhat fortuitous and at the same time deceptive coincidence as other exponents come out incorrectly, and the actual value of the fractal dimension is close to $2, D \approx 1.96$ [31]. Describing the critical behaviour of an interacting Bose gas, using quasiparticles with the spectrum

$$
E(k) \sim k^{3 / 2},
$$

as was done by Baym et al. [33], can therefore not be justified.

In addition to numerical studies of the static, classical theory, the coefficient $C$ has also been estimated by analytical studies of that theory (for a summary, see Ref. [1]), such as the $1 / N$ expansion [34] and variational perturbation theory [35].

Although the dynamic modes do not, due to cancellations, contribute to the shift in the condensation temperature linear in $a$, they do determine the coefficient of the next term $\sim a^{2} n^{2 / 3} \ln \left(a n^{1 / 3}\right)$ in that shift [5]. 


\section{A. M. J. SCHAKEL}

\section{CONCLUSIONS}

In this paper, the effect of fluctuations in the nonzero frequency modes of a weakly interacting Bose gas on the condensation temperature was studied in detail. The simple algorithm used to perturbatively calculate the effect in a high-temperature expansion in conjunction with zeta function regularization of infrared divergences was demonstrated to be reliable by showing that a host of known results are recovered. The presence of temperature-induced energy gaps for these dynamic modes were argued to allow for a perturbative approach. It was shown that the shift in the condensation temperature of the form (92) with

$$
C=-\frac{8}{3} \frac{\zeta\left(\frac{1}{2}\right)}{\zeta^{1 / 3}\left(\frac{3}{2}\right)} \approx 2.83,
$$

we had obtained earlier at one loop [3] is canceled by two-loop contributions [4].

\section{ACKNOWLEDGMENTS}

The author acknowledges helpful correspondence with and critical comments of P. Arnold, M. Haque and H. Kleinert.
[1] G. Baym, J.-P. Blaizot, M. Holzmann, F. Laloë, D. Vautherin, Eur. J. Phys. B 24, 104 (2001).

[2] S. Singh, P. N. Pandita, Phys. Rev. A 28, 1752 (1983).

[3] A. M. J. Schakel, Int. J. Mod. Phys. B 8, 2021 (1994).

[4] This cancellation was pointed out to the author by P. Arnold, see also Ref. [5].

[5] P. Arnold, G. Moore, B. Tomášik, Phys. Rev. A 65, 013606 (2001).

[6] A. L. Fetter, J. D. Walecka, Quantum Theory of ManyParticle Systems, (McGraw-Hill, New York, 1971).

[7] L. Dolan, R. Jackiw, Phys. Rev. D 9, 3320 (1974).

[8] K. Huang, Statistical Mechanics (Wiley, New York, 1987).

[9] R. J. Rivers, Path Integrals in Quantum Field Theory (Cambridge University Press, Cambridge, 1987), Sec. 14.3 .

[10] S. Bund, A. M. J. Schakel, Mod. Phys. Lett. B 13, 349 (1999).

[11] R. P. Feynman, Phys. Rev. 90, 1116 (1953); ibid. 91, 1291 (1953).

[12] J. D. Gunton, M. J. Buckingham, Phys. Rev. 166, 152 (1968); see also the textbook: R. K. Pathria, Statistical Mechanics, 2nd edition (Butterworth-Heinemann, Oxford, 1996), Sec. 12.5.

[13] G. 't Hooft, M. Veltman, Diagrammar (CERN Yellow Report 73-9, 1973).

[14] J. E. Robinson, Phys. Rev. 83, 678 (1951); F. London, Superfluids (Wiley, New York, 1954), Vol. 2, Appendix.

[15] H. E. Haber, H. A. Weldon, Phys. Rev. Lett. 46, 1497 (1981); Phys. Rev. D 25, 502 (1982).

[16] J. I. Kapusta, Finite Temperature Field Theory, (Cambridge University Press, Cambridge, 1989).

[17] M. Abramowitz, I. A. Stegun, Handbook of Mathematical Functions, (Dover, New York, 1972).

[18] L. P. Gorkov, Sov. Phys. JETP 9, 1364 (1959).

[19] R. P. Feynman, H. Kleinert, Phys. Rev. A 34, 5080
(1986).

[20] H. Kleinert, Path Integrals in Quantum Mechanics, Statistics and Polymer Physics, 2nd Edition, (World Scientific, Singapore, 1995), Ch. 5.

[21] N. N. Bogoliubov, J. Phys. USSR 11, 23 (1947).

[22] A. M. J. Schakel, Boulevard of Broken Symmetries, Habilitationsschrift, Freie Universität Berlin, cond-mat/9805152 (1998).

[23] N. M. Hugenholtz, Rep. Prog. Phys. 28, 201 (1965).

[24] A. A. Abrikosov, L. P. Gorkov, I. E. Dzyaloshinski, Methods of Quantum Field Theory in Statistical Physics (Prentence-Hall, Englewood Cliffs, 1963).

[25] N. M. Hugenholtz, D. Pines, Phys. Rev. 116, 489 (1959).

[26] D. S. Fisher, P. C. Hohenberg, Phys. Rev. B 37, 4936 (1988).

[27] V. N. Popov, Functional Integrals in Quantum Field Theory and Statistical Physics (Reidel, Dordrecht, 1983), Ch. 6.

[28] D. I. Uzunov, Phys. Lett. A 87, 11 (1981); Introduction to the Theory of Critical Phenomena (World Scientific, Singapore, 1993).

[29] P. Grüter, D. Ceperly, F. Laloë, Phys. Rev. Lett. 79, 3549 (1997).

[30] P. Arnold, G. Moore, Phys. Rev. Lett. 87, 120401 (2001); V. A. Kashurnikov, N. Prokof'ev, and B. Svistunov, ibid. 120402 (2001).

[31] A. M. J. Schakel, Phys. Rev. E 63, 026115 (2001); J. Low Temp. Phys 129, 323 (2002).

[32] J. A. Lipa et al., Phys. Rev. Lett. 84, 4894 (2000).

[33] G. Baym, J.-P. Blaizot, M. Holzmann, F. Laloë, D. Vautherin, Phys. Rev. Lett. 83, 1703 (1999).

[34] G. Baym, J.-P. Blaizot, J. Zinn-Justin, Europhys. Lett. 49, $150(2000)$.

[35] H. Kleinert, Five-Loop Critical Temperature Shift in Weakly Interacting Homogeneous Bose-Einstein Condensate, cond-mat/0210162 (2002). 


\title{
РЕГУЛЯРИЗАЦІЯ ІНФРАЧЕРВОНИХ РОЗБІЖНОСТЕЙ У КОНДЕНСАЩЇ̈ БОЗЕ-АЙНШТАӤНА ЗА ДОПОМОГОЮ $\zeta$-ФУНКЦЇ̈
}

\begin{abstract}
А. Шакель
Інститут теоретичной фізики

Арнім-аллее, 14, Берлін, 14195, Німеччина

Зроблено огляд обчислення методом теорії збурень впливу флуктуацій на температуру конденсації у ненульових частотних модах слабковзаємодіючого бозе-газу. Ці динамічні моди, якими нехтують у більшості сучасних досліджень, мають температурно-спричинену енергетичну щілину, що виправдовує пертурбативний підхід. Опрацьовано простий, але потужний алгоритм для розрахунку цього ефекту при високотемпературному розвиненні в поєднанні з регуляризацією інфрачевоних розбіжностей за допомогою $\zeta$-функції. Цей алгоритм виявляється надійним, оскільки він відтворює відомі результати для низки прикладів. При врахованих двопетлевих внесках показано, що динамічні моди не спричиняють зсуву в температурі конденсащії. Це дає підстави переглянути наші попередні результати, отримані для однієї петлі.
\end{abstract}

